\title{
Inbreeding depression drives evolution of dispersal and polyandry
}

\author{
Greta Bocedi ${ }^{1}$
}

${ }^{1}$ School of Biological Sciences, University of Aberdeen, Zoology Building, Tillydrone Avenue, Aberdeen, AB24 2TZ, UK.

\section{Abstract}

Inbreeding depression, defined as the reduction in fitness components of offspring of related individuals compared to offspring of unrelated individuals, is a widespread phenomenon and has profound demographic and evolutionary consequences. It can reduce the mean fitness of a population and increase extinction risk, and it can affect traits evolution. Inbreeding depression is widely hypothesized to be a key driver of the evolution of, among other traits, dispersal (individual movements potentially leading to spatial gene flow) and polyandry (female mating with multiple males within a single reproductive bout), as mechanisms to avoid inbreeding. In turn, both dispersal and polyandry can change the relatedness structure within and among populations, thus affecting opportunity for inbreeding and consequent evolution of inbreeding depression. However, despite this potential major shared driver, and despite the large amount of both theoretical and empirical work, evolution of dispersal and polyandry given inbreeding have been so far studied separately, and thus we still do not know whether and how dispersal and polyandry affect each other's evolution, and how they may feed-back onto evolution of inbreeding depression itself. Here, using a genetically-explicit individual-based model, which models realistic distributions of selection and dominance coefficients of deleterious recessive mutations underpinning inbreeding depression, I show that: 1) inbreeding depression indeed drives evolution of dispersal and polyandry; 2) there is a negative feedback between dispersal evolution and polyandry evolution, which therefore evolve as alternative inbreeding avoidance strategies; 3) inbreeding depression is mainly shaped by the level of dispersal, while polyandry has a much more limited effect.

Key words: female multiple mating, dispersal, heterosis, deleterious recessive mutations, genetically explicit modelling 


\section{Introduction}

Inbreeding depression, defined as the reduction in fitness components of offspring of related individuals compared to offspring of unrelated individuals, is a widespread phenomenon and has profound demographic and evolutionary consequences (Keller and Waller 2002; Charlesworth and Willis 2009). It can reduce the mean fitness of a population and increase extinction risk (Theodorou and Couvet 2006; Hedrick and Garcia-Dorado 2016), and it can affect trait evolution (Lande and Schemske 1985; Charlesworth and Charlesworth 1987; Szulkin et al. 2013). Inbreeding depression is widely hypothesized to be a key driver of the evolution of, among other traits, dispersal (i.e., any individual movement potentially leading to spatial gene flow (Ronce 2007; Clobert et al. 2012)), and polyandry (i.e., female mating with multiple males within a single reproductive bout (Pizzari and Wedell 2013; Taylor et al. 2014)), as mechanisms to avoid inbreeding (Waser et al. 1986; Perrin and Mazalov 1999; Jennions and Petrie 2000; Tregenza and Wedell 2002). In turn, both dispersal and polyandry can change the relatedness structure within and among populations, thus affecting opportunity for inbreeding and consequent evolution of inbreeding depression. However, despite this potential major shared driver, and despite the large amount of both theoretical and empirical work, evolution of dispersal and polyandry given inbreeding have been so far studied separately, and thus we still do not know whether and how dispersal and polyandry affect each other's evolution, and how they may feed back onto evolution of inbreeding depression itself. Filling this knowledge gap is particularly important because populations exist in space and it is unlikely that dispersal and mating system evolve independently. Further, ongoing environmental changes, such as habitat fragmentation and isolation, are fragmenting populations in smaller demes thus increasing the risk of inbreeding. On the other hand, habitat fragmentation and isolation may increase the cost of dispersal (Bonte et al. 2012; Cote et al. 2017; Legrand et al. 2017). The resulting balance between inbreeding depression, dispersal costs and changed population genetic structure, might therefore drive further evolution of dispersal and/or polyandry in the face of ongoing environmental changes. 
It is now accepted that inbreeding depression and its converse, heterosis (i.e., the increase in fitness in offspring originating from between populations crosses relative to offspring from within population crosses; (Charlesworth and Charlesworth 1987; Whitlock et al. 2000; Charlesworth and Willis 2009)) can drive dispersal evolution, although debate remains on the form and the relative importance of this effect (Perrin and Goudet 2001; Ronce 2007). Theoretical work has shown inbreeding depression and heterosis can results in substantial evolution of dispersal, which may be sex-biased or equal between the sexes depending on factors such as the cost of dispersal, the type and strength of same sex competition, the mating system (e.g., monogamy vs polygyny), the strength of inbreeding depression and the presence of demographic and/or environmental stochasticity (Gandon 1999; Perrin and Mazalov 2000; Guillaume and Perrin 2006, 2009; Roze and Rousset 2009; Henry et al. 2016; Li and Kokko 2019). Substantial insights have been achieved by theoretical models that consider the joint evolution of dispersal and inbreeding depression. These models do not assume constant inbreeding depression but explicitly model the accumulation and purging of deleterious recessive mutations responsible for inbreeding depression and genetic load more generally (Guillaume and Perrin 2006, 2009; Roze and Rousset 2009; Henry et al. 2016). Particularly, Roze and Rousset (2009) by using a continuous chromosome model, which allows the modelling of a potentially infinite number of deleterious recessive mutations, showed that heterosis can have a much more important effect (relative to kin competition) on dispersal evolution than previously thought (Guillaume and Perrin 2006; Ravigné et al. 2006), especially when population size is large and the genomic deleterious mutation rate is in the upper range of observed values. Further, the effect of heterosis increases when mutations become more recessive (Guillaume and Perrin 2006; Roze and Rousset 2009). However, even studies that include a genetically explicit model of inbreeding depression, generally assume a fixed selection coefficient, $s$, and a fixed dominance coefficient, $h$, across deleterious mutations. Thus, we still do not know how a more realistic distribution of deleterious mutations, which likely comprises many mutations with very small fitness effect and rare ones with larger effect (Eyre-Walker and Keightley 2007), and a negative relationship 
between selection and dominance coefficients (Agrawal and Whitlock 2011; Huber et al. 2018), might impact on evolving inbreeding depression, and the consequent evolution of dispersal and mating systems (Porcher and Lande 2016).

Less established, in its theoretical and empirical demonstration, is the hypothesis that inbreeding depression can drive evolution of female multiple mating, especially when there is direct selection against it, that is, when polyandry is costly to females (Reid and Sardell 2012; Reid et al. 2015; Duthie et al. 2016; Germain et al. 2018). Polyandry has been hypothesized to evolve as a mechanism for inbreeding avoidance through two main routes: direct or indirect inbreeding avoidance (Germain et al. 2018). Female multiple mating could evolve because it facilitates inbreeding avoidance through female active pre- and/or post-copulatory allocation of paternity to less closely related males, hence directly reducing inbreeding depression in offspring viability (direct inbreeding avoidance) (Jennions and Petrie 2000; Tregenza and Wedell 2002; Duthie et al. 2016, 2018). Alternatively, without invoking active mate choice and kin recognition, polyandry could evolve because it alters relatedness among a female's offspring, producing more half-sibs rather than full-sibs, thereby reducing the risk of close inbreeding for the offspring of a polyandrous female and reducing inbreeding depression in her grand offspring (indirect inbreeding avoidance) (Cornell and Tregenza 2007; Germain et al. 2018). Few theoretical models have investigated these verbal predictions (Cornell and Tregenza 2007; Duthie et al. 2016, 2018) and generally concluded that the strength of indirect selection on polyandry through inbreeding avoidance might be very small, thereby suggesting a minor role of inbreeding depression in polyandry evolution.

Particularly, Cornell and Tregenza (2007) concluded that purging of deleterious recessive mutations makes it unlikely to maintain sufficient levels of inbreeding depression to favour costly polyandry, and that evolution of polyandry as a mechanism of indirect inbreeding avoidance is far more likely if inbreeding depression was due to overdominance. This poses a problem because current understanding suggests that inbreeding depression is 
predominantly caused by deleterious recessive mutations rather than by overdominant mutations (Charlesworth and Willis 2009). However, Cornell and Tregenza's model (Cornell and Tregenza 2007) makes some assumptions that preclude assessing whether such a mechanism could generally drive polyandry evolution in spatially structured populations. The model considers alternating generations of outbreeding and inbreeding, which is particularly relevant to some invertebrate groups where mated females cyclically colonize empty patches, thus experiencing cyclical changes in inbreeding risk (e.g., store product pest species such as flour beetles, Tribolium spp.). However, this does not apply to species with more regular inbreeding as it does not consider the building up of complex relatedness structure within a population that arises across multiple generations and might weaken the selective advantage of polyandry (Germain et al. 2018). Indeed, Cornell and Tregenza's work (Cornell and Tregenza 2007) pointed out that higher levels of inbreeding, as we might expect in spatially structured populations, might favour polyandry, although this might be offset by greater purging of deleterious recessives alleles. No model so far has investigated the evolution of polyandry as a mechanism of indirect inbreeding avoidance in populations that are spatially structured and connected by dispersal, nor has any considered an explicit model of inbreeding depression with realistic distributions of the fitness effects and dominance coefficients of deleterious mutations, thus leaving a substantial knowledge gap.

Beyond the unknowns that are still present in the separate theories of dispersal and polyandry evolution given inbreeding depression, we do not know how these two potential mechanisms of inbreeding avoidance might affect each other's evolution, and feed back onto evolution of inbreeding depression. I hypothesize a negative feedback between dispersal and polyandry, whereby the evolution of polyandry might reduce inbreeding and hence reduce the strength of selection for dispersal and, vice versa, the presence of high dispersal might weaken selection for polyandry. The outcome of this tug-o-war will likely depend on the relative 
would be much more effective, on the level of inbreeding load, and on the strength of direct selection against them, i.e., on the cost of dispersal and polyandry.

Here, I investigate this hypothesis with a modelling framework that allows joint evolution of dispersal and polyandry in spatially structured populations and, at the same time, explicit accumulation of deleterious mutations and evolution of inbreeding depression. Specifically, inbreeding depression is determined by the accumulation of a potentially infinite number of deleterious recessive mutations with a realistic distribution of fitness effects and dominance coefficients. First, I test whether existing predictions on the independent evolution of dispersal and polyandry hold in spatially structured populations given an explicit model of inbreeding and inbreeding depression evolution, and how their evolution affects inbreeding depression. Second, I test the novel hypothesis of a negative feedback between jointly evolving dispersal and polyandry and, third, determine their joint effect on the evolution of inbreeding depression.

\section{The Model}

To investigate the joint evolution of dispersal and polyandry given inbreeding depression I built a spatially and genetically explicit individual-based model where emigration probability $(d)$ and female re-mating rate (a) evolve. Mutation load and inbreeding depression (ID) also evolve by accumulation and purging of deleterious recessive mutations. Populations of a dioecious species, with non-overlapping generations, occupy cells within a landscape grid of 20 by 20 cells, and are connected by dispersal. The environment is spatially homogeneous and temporally constant; each cell is suitable to hold a population with constant carrying capacity $K=50$. All the model variables and parameters are listed in Table S1.

Genetic architecture and inbreeding depression. Individuals carry two unlinked diploid loci with a continuous distribution of alleles (Kimura 1965) underlying $d$ and a respectively. The initial value of each allele is sampled from normal distributions. Alleles can mutate with 
probability $\mu=10^{-3} /$ allele/generation. When a mutation occurs a random normal deviate with mean zero is added to the allele value. The individual's genotypic values for $d$ and $a$ ( $g_{d}$ and $g_{a}$ ) are given by the sum of the two allelic values at the respective loci. The phenotypic expression has no environmental variance and is female limited for a. I assume the phenotypes $a \geq 0$ and $0 \leq d \leq 1$.

ID is determined by deleterious recessive mutations (Charlesworth and Willis 2009) which accumulate on a continuous chromosome (Roze and Rousset 2009). Each individual carry two homologous autosomes of length $R$ (genome map length). The position of each new deleterious mutation on the chromosome is sampled from the continuous uniform distribution $U[0, R]$. The number of loci at which mutations can occur is therefore effectively infinite ("infinite site model"; Peischl et al. 2015). Each new mutation is characterized by a selection coefficient $s$, determining the mutation's effect in the homozygous state, and a dominance coefficient $h$. The effect of each mutation $i$ is multiplicative such that the genetic fitness $\omega$ of an individual is given by

$$
\omega=\prod_{\text {Nhet }}\left(1-h_{i} s_{i}\right) \prod_{\text {Nhom }}\left(1-s_{i}\right)
$$

Nhet and Nhom represent the number of heterozygous and homozygous mutations respectively. Deleterious mutations are of two types: mildly deleterious and lethal (Gilbert et al. 2017; Spigler et al. 2017). Mildly deleterious mutations occur at a rate $U_{d}=1.0 /$ diploid genome/generation. The selection coefficient of each new mutation is sampled from a gamma distribution with mean $S_{d}=0.05$ and shape parameter $\alpha=1$ (Schultz and Lynch 1997; Spigler et al. 2017). The dominance coefficient of a mutation $i$ depends on its selection coefficient $s_{i}$ and is sampled from the continuous uniform distribution $U\left[0.0, e^{-k s_{i}}\right] . k$ is defined as $-\log \left(2 h_{d}\right) / s_{d}$, where $h_{d}$ is the mean dominance coefficient $\left(h_{d}=0.3\right)$ (Caballero and Keightley 1994; Spigler et al. 2017). Lethal mutations occur at rate $U_{l}=0.2 /$ diploid genome/generation and are extremely recessive, with constant selection coefficient $s_{l}=1$ and 
dominance coefficient $h_{l}=0.02$ (Simmons and Crow 1977; Lande et al. 1994; Porcher and Lande 2005; Spigler et al. 2017). At each generation, the number of new mutations per diploid genome is sampled from Poisson distributions with parameters $U_{d}$ and $U_{l}$. The number of crossovers along the continuous chromosomes is sampled from a Poisson distribution with mean $R$, and the position of each crossover is sampled from the uniform distribution $U[0, R]$.

Individuals also carry $L_{n}=500$ neutral autosomal diploid loci to determine the degree to which individuals are inbred (Bocedi and Reid 2017). Neutral allelic values are continuously distributed, sampled from the uniform distribution $U[-1000.0,1000.0]$, and mutate with probability $10^{-3} /$ allele/generation. Neutral loci recombine at rate $r=0.1$. Alleles at the same locus will be identical only by descent as the chance of non-descent identity by state, stemming from initialization or mutation, is negligible. For this reason, an individual's neutral homozygosity, defined as the number of neutral homozygous loci / $L_{n}$, represents a proxy for the realized individual coefficient of inbreeding, hereafter noted as $F_{\text {homoz }}$ (Markert et al. 2004; Neff and Pitcher 2008; Fromhage et al. 2009; Bocedi and Reid 2017). When an individual is born, it is assumed to die immediately if its genetic fitness $\omega=0$. If $\omega>0$, the new-born survives to adulthood (unless it incurs in dispersal mortality, see below) when $\omega$ will determine its probability of reproducing. Thus, ID is affecting two fitness components: 1) reduction in offspring survival, which is determined by the presence of homozygous lethal mutations, and 2) reduction in adult probability of reproducing, which is determined mainly by mildly deleterious mutations.

The level of ID present in a metapopulation at a given point in time, was calculated as: 1) effect of $F_{\text {homoz }}$ on offspring survival by fitting a generalized linear model with Poisson distribution and logarithmic logit link function (Nietlisbach et al. 2019); 2) effect of $F_{\text {homoz }}$ on the logarithm of adult reproduction probability by fitting a linear model (Morton et al. 1956). All models were fitted in R (R Core Team 2019). To obtain a spread of $F_{\text {homoz }}$ values to estimate ID, I created individuals by selecting the central 140 populations in the landscape (out of 400 
populations) at a given point in time. For each of these populations, I mated each female with 10 males selected randomly within the female's population and with 10 males selected randomly between different populations. Each mating produced one offspring. Models were then fitted on all the offspring pooled together. All the offspring produced this way were used only for estimating ID and then discarded, thus were not part of the ecological and evolutionary dynamics.

Life cycle and selection. At each generation, the life cycle consists in reproduction (mating and offspring birth), adults' death and death of offspring with $\omega=0$, offspring dispersal and density-dependent survival. An adult's probability of reproducing is given by its genetic fitness $\omega$. Each reproducing female $i$ mates initially once, and then re-mates with probability Pmati depending on her re-mating rate phenotype $a_{i}$ and on her current number of mates $N m_{i}$ :

$$
\operatorname{Pmat}_{i}=e^{-a_{i} N m_{i}}
$$

Each mate is randomly sampled among the reproducing males in the female's population, without replacement. If the female has already mated once with all the reproducing males in the population, she stops re-mating. Mating multiply can be costly to females. The probability that a female $i$ survives to reproduction $\left(\psi_{i}\right)$ depends on her total number of mates Nmates $i$ and on the strength of selection against multiple mating $\omega_{m}^{2}$ :

$$
\psi_{i}=e^{-\frac{\left(1-N \text { mates }_{i}\right)^{2}}{2 \omega_{m}^{2}}}
$$

If the female survives mating, she produces a number of offspring sampled from a Poisson distribution with mean $f=12$ and primary sex-ratio $=1: 1$. Each offspring is sired by a male randomly chosen, with replacement, between the female's mates.

After reproduction, all adults die, and offspring may disperse among sub-populations according to their emigration probability phenotype $d$. Dispersal distance and direction are 
sampled from a negative exponential distribution (mean 2 cells) and uniform distribution between 0 and $2 \pi$ respectively. The new location is re-sampled if it falls outside the grid. Dispersal has a cost, $c_{d}$, representing the probability of an individual dying during dispersal. After dispersal, density-dependent survival takes place in each population. Individuals survive with probability $\min (K / N, 1)$, where $N$ is the total number of individuals in the population.

Simulations. I ran three main sets of simulations. In the first set, only dispersal was evolving while female re-mating rate was constant, $a=3$ (corresponding to 1.3 mates per female on average - hereafter defined as monandry). In the second set, only female re-mating rate was evolving while dispersal was constant, $d=0.05$. In the third set, both dispersal and female remating rate were evolving. All simulations were run under varying costs of dispersal and female re-mating and repeated in the absence of deleterious mutations (and hence ID) as control. Additionally, I tested the effect of varying the deleterious mutation rates.

\section{Results and Discussion}

Evolution of dispersal and inbreeding depression given monandry. Under fixed monandry conditions, emigration probability $d$ evolved in response to the presence of deleterious recessive mutations and consequent inbreeding depression (ID), reaching higher values the lower the survival cost of dispersal $\left(c_{d}\right.$; Fig. 1A). The evolved emigration probability in the presence of deleterious mutations was much higher compared to when genetic load was absent from the model and the only driver of dispersal evolution was kin competition, given the spatio-temporally homogeneous environment. For example, at the lower cost considered $\left(c_{d}=0.3\right), d$ evolved to be almost eight times higher with ID [median $\bar{d}=0.41$ (95\% Cl 0.401-0.414), where $\bar{d}$ represents the mean phenotypic value for one replicate metapopulation; median and $\mathrm{Cl}$ are taken across 20 replicates] than without ID [median $\bar{d}=$ $0.05(95 \% \mathrm{Cl} 0.05-0.054)]$, while it evolved to almost seven times higher at high cost of dispersal [ $c_{d}=0.6$; median $\bar{d}=0.15(95 \% \mathrm{Cl} 0.15-0.157)$ with ID vs. median $\bar{d}=0.02(95 \% \mathrm{Cl}$ 0.02-0.023) without ID]. Results remained qualitatively similar under lower rates of deleterious 
mutations, although the lower the mutation rate the lower the evolved dispersal probability (Fig. S1A-S2A). The level of heterosis (i.e., the converse of ID) reached at equilibrium depended on the level of evolved dispersal, and hence on dispersal cost (Fig. S3A). Higher costs of dispersal led to lower dispersal and hence to populations being less homogeneous in terms of their genetic load. This led to higher heterosis at higher dispersal costs. Higher rates of deleterious mutations, by causing accumulation of higher genetic load, led to the emergence of higher heterosis compared to lower mutation rates. These results broadly agree with the conclusions of Roze and Rousset (Roze and Rousset 2009) who, by using a genetically explicit, infinite site model of genetic load, show that heterosis can have a substantial role in dispersal evolution, in contrast with what shown by previous models (Guillaume and Perrin 2006; Ravigné et al. 2006).

The level of ID reached at equilibrium depended on the level of evolved dispersal, and hence on dispersal cost (Fig. 1C-E; Table S2). Higher dispersal cost led to lower $d$ and consequent higher inbreeding within local populations, reflected by higher neutral homozygosity (Fig. 1C). In turn, more inbreeding facilitated purging of deleterious recessive mutations thus reducing ID. This was true for both component of ID. Both lethal mutations (strongly recessive mutations causing ID in early life - offspring survival, Fig. 1E) and mildly deleterious mutations (causing ID in later life - adult reproduction probability, Fig. 1C) experienced a greater purging at lower dispersal, where both the mutation load (i.e., the decrease in fitness for outbred individuals) and ID were lower compared to scenarios with much higher dispersal evolving, albeit grater differences were observed for ID rather than for mutation load (Table S2). This pattern was conserved at lower mutation rates although much less genetic load accumulated, leading to higher fitness and lower mutation and inbreeding load (Fig. S1C-E; S2C-E). 

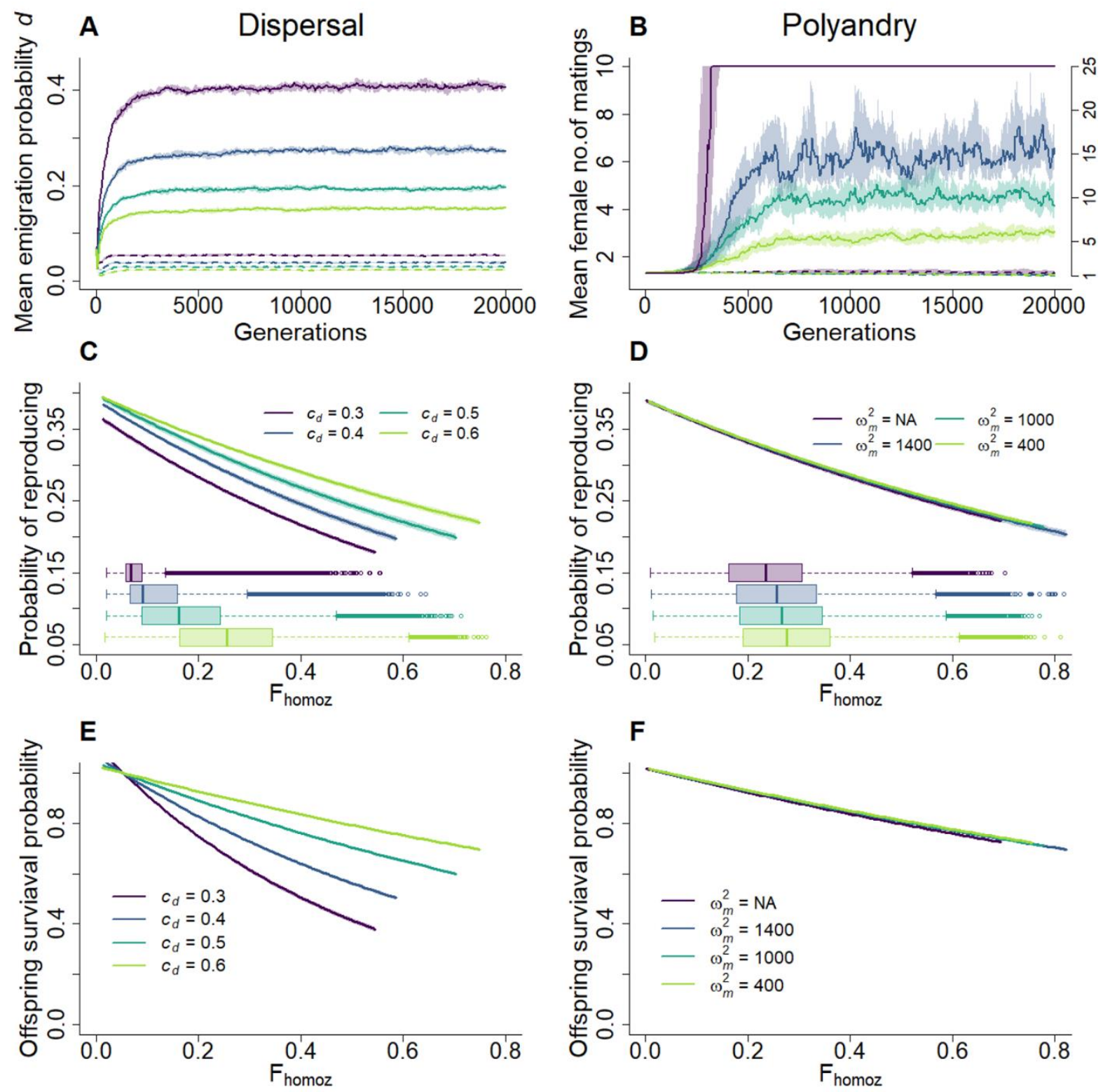

Figure 1. Inbreeding depression promotes evolution of dispersal and polyandry when either one trait or the other evolves. A) Evolution of mean dispersal probability phenotypes $d$ in the absence of polyandry $(a=3.0)$, under different costs of dispersal $\left(c_{d}=0.3,0.4,0.5\right.$, 0.6 ), in the absence (i.e., no ID; dashed lines) or presence (solid lines) of deleterious mutations. B) Evolution of mean polyandry phenotypes (mean female number of matings $=1$ $+1 / a)$ under fixed dispersal probability $(d=0.05)$, as a function of different strengths of direct selection against female remating (no cost; $\omega_{m}^{2}=1400,1000,400$ ) in the absence (dashed lines) or presence (solid lines) of ID. In the presence of deleterious mutations and no direct selection against polyandry $\left(\omega_{m}^{2}=\mathrm{NA}\right)$, females evolve to mate with all the males in the population; the $y$-axis on the right hand-side refers to this single line. Lines represent the median of mean phenotypes across 20 replicated simulations; coloured shades depict the first and third quartile. C-D) Relationship between individual probability of reproducing and inbreeding coefficient $F_{\text {homoz }}$ (i.e., ID in reproduction probability) when $\mathrm{C}$ ) dispersal evolves 
under different costs, in the absence of polyandry and, D) polyandry evolves under different strengths of direct selection, with fix dispersal probability. Lines show the fitted models and coloured shades the $95 \% \mathrm{Cl}$. Models are fitted to simulations results at generation 20,000 to a subsample of 140 populations, across 10 replicates. Boxplots represents the distribution of the individual $F_{\text {homoz. }}$ E-F) Relationship between offspring survival probability and $F_{\text {homoz }}$ (i.e., ID in offspring survival probability). In E) simulation scenarios and parameters as in C); in F) as in D). The coefficients (i.e., mutation load and inbreeding load) and standard errors of all the fitted models are presented in Table S2.

Evolution of polyandry and inbreeding depression given low dispersal. Like dispersal, polyandry (female number of matings, $P=1+1 / a$ ) evolved in response to ID when dispersal was low and not evolving, to a level that depended on the fitness cost of female multiple mating (Fig. 1B; Fig. S4). Given no cost of polyandry, females evolved to mate with all the males present in the population. However, even a very small cost reduced substantially $\bar{P}$ (where $\bar{P}$ represents the mean phenotypic value for one replicate metapopulation); yet moderate polyandry evolved. For example, a strength of direct selection on re-mating $\omega_{m}^{2}=1000$ led to evolution of median $\bar{P}=4.13(95 \% \mathrm{Cl} 3.51-5.26)$ (median and $\mathrm{Cl}$ are taken across 20 replicates), corresponding to an average realized survival cost of 0.005 for females. On the contrary, in the absence of ID, polyandry did not evolve even when free of cost (Fig. 1B and S4, dashed lines). Lower rates of mildly deleterious and lethal mutations $\left(U_{d}=0.5\right.$ and $U_{I}=$ $0.1 ; U_{d}=0.1$ and $U_{l}=0.02$ ) substantially reduced selection for polyandry such that hardly any polyandry evolved, or evolution took a considerable time (Fig. S1B; S2B).

These results provide a positive answer to the still standing question of whether costly polyandry can evolve, at least in theory, as a mechanism of indirect inbreeding avoidance (Germain et al. 2018). Specifically, they show that costly polyandry, although the cost needs to be quite low, can indeed evolve in response to inbreeding depression given: i) inbreeding depression caused exclusively by deleterious recessive mutations, and without the need for overdominant mutations (Cornell and Tregenza 2007); ii) realistic deleterious mutation rates and distribution of fitness effects; iii) and complex sibship structure emerging in spatially 
structured populations (Germain et al. 2018). Thus, polyandry evolution as means of indirect inbreeding avoidance might be more widespread than previously thought (Cornell and Tregenza 2007; Germain et al. 2018), and therefore a potentially important mechanism to explain the existence of even low levels of polyandry across multiple systems, especially when dispersal is low.

Although polyandry evolved in response to the presence of genetic load, the level of evolved polyandry under different costs did not substantially change the level of inbreeding (measured as neutral homozygosity) and ID at equilibrium (Fig. 1D-F; Table S2; Fig. S1D-F; S2D-F). In fact, both the mutation load and ID were the same across levels of evolved polyandry and polyandry's costs. The level of mutation and inbreeding load was mainly determined by dispersal, which was fixed to $d=0.05$, and similar to that which evolved under high cost of dispersal and no polyandry (Fig. 1C-E). This was true also for heterosis (Fig. S4). Thus, under low fix dispersal, polyandry seems to have a minimal effect, if any, on the genetic load, ID and heterosis.

Joint evolution of dispersal, polyandry, and inbreeding depression. When dispersal and polyandry could evolve jointly, results clearly confirmed the hypothesis of a negative feedback between two complementary mechanisms of inbreeding avoidance (Fig. 2; S5). This feedback was modulated by the relative costs of the two evolving behaviours. For a given cost of dispersal $\left(c_{d}\right)$, higher dispersal evolved under monandry; vice versa, the higher the evolved level of polyandry (hence for lower costs of female re-mating) the lower the evolved emigration probability. For example, $c_{d}=0.3$ led to evolution of median $\bar{d}=0.39(95 \% \mathrm{Cl} 0.375-0.396)$ and median $\bar{P}=1.97(95 \% \mathrm{Cl} 1.763-2.173)$ for high cost of female re-mating $\left(\omega_{m}^{2}=400\right)$, while it led to median $\bar{d}=0.31(95 \% \mathrm{Cl} 0.312-0.322)$ and to females mating with all the males in the population $(P=25)$ when female re-mating did not carry costs. On the other hand, for a given cost of female re-mating, higher polyandry evolved given lower evolved dispersal (hence for higher $\left.c_{d}\right)$. For example, $\omega_{m}^{2}=1000$ led to evolution of median $\bar{d}=0.13(95 \% \mathrm{Cl} 0.126-0.131)$ 
and median $\bar{P}=4.49(95 \% \mathrm{Cl} 3.842-5.317)$ for high cost of dispersal $\left(c_{d}=0.6\right)$, while it led to median $\bar{d}=0.38(95 \% \mathrm{Cl} 0.36-0.383)$ and median $\bar{P}=2.58(95 \% \mathrm{Cl} 2.467-2.814)$ for $C_{d}=0.3$. while polyandry did not evolve.
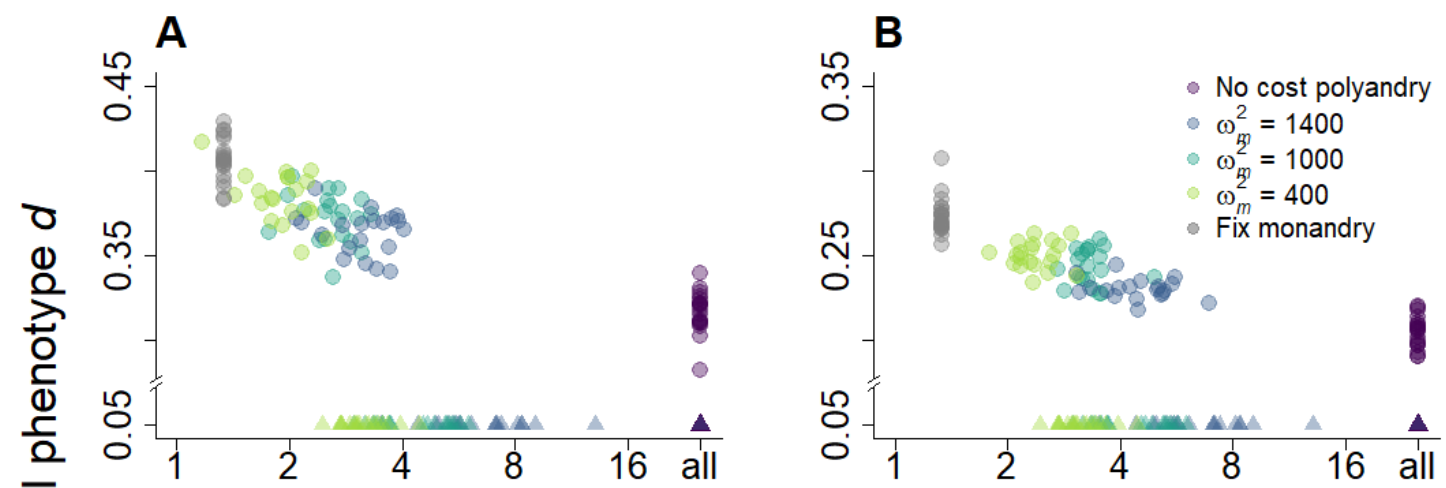

C

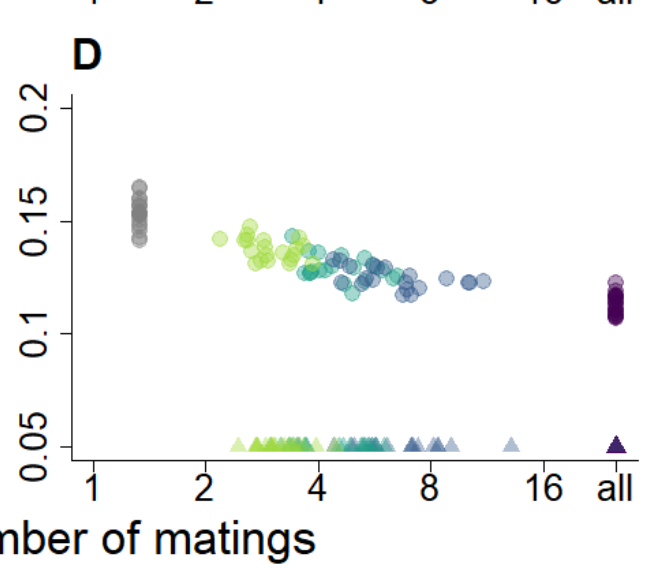

Figure 2. Dispersal and polyandry negatively feedback to each other evolution under inbreeding depression. Joint evolution of mean dispersal probability phenotypes $d$ and mean polyandry phenotypes (mean female number of matings: $P=1+1 / a$ ) in the presence of inbreeding depression, given different costs of dispersal $C_{d}(\mathbf{A}: 0.3 ; \mathbf{B}: 0.4 ; \mathbf{C}: 0.5 ; \mathbf{D}: 0.6)$ and different strengths of direct selection against female re-mating (no cost; $\omega_{m}^{2}=1400,1000,400$ ). Each data point represents the mean phenotypic values for one out of 20 replicate simulations at generation 20,000. Coloured dots indicate simulations where dispersal and polyandry jointly evolved; triangles, simulations where polyandry evolved given fix dispersal probability $(d=$ $0.05)$; grey dots, simulations where dispersal evolved given fix monandry $(a=3.0)$. The $x$-axis 372 is on the logarithmic scale to aid visualization. Note the different $y$-axis scales for the four panels. Other parameters: $U_{d}=1.0, U_{l}=0.2$. 

was present at the metapopulation level (i.e., simulation replicate level), whereby for a given cost of dispersal and strength of direct selection on female re-mating, systems that evolved higher mean emigration probability phenotypes, also evolved lower mean female number of matings, and vice versa (Fig. S7). This correlation was present when female re-mating was costly; in the absence of re-mating costs this correlation disappeared as females consistently evolved to mate with all the males in the population. Within a metapopulation, there was no evidence of a negative correlation between $d$ and $P$, whereby subpopulations with higher mean dispersal might have had lower mean polyandry (Fig. S8). Further, there was no evidence of any genetic correlation between $g_{d}$ and $g_{a}$, nor between $d$ and $P$, thus excluding the possibility of indirect selection between the two traits (Fig. S9). dispersal and polyandry (Fig. S10), similarly to when the two traits evolved independently (Fig. S1-S2). Polyandry hardly evolved, apart from few exception simulations under no or weak direct selection against female re-mating, where it reached high levels. Strong negative correlation between dispersal and polyandry phenotypes was present at the metapopulation level when some polyandry evolved, while it disappeared for very low mutation rates combined with costly polyandry (Fig. S11). As for higher rates of deleterious mutations, at lower mutation rates there was no evidence of a negative correlation between dispersal and polyandry at the subpopulation level, nor of any genetic correlation (Fig. S12-S13).

The evolved level of ID depended mainly on the cost of dispersal, and hence on the level of evolved dispersal (Fig. 3-4; Table S3). As for the scenario where dispersal evolved under fix monandry (Fig. 1C-E), higher cost and consequent lower evolved dispersal led to lower inbreeding depression to both probability of reproducing (Fig. 3A, Fig. 4 diamonds) and offspring survival (Fig. 3B, Fig. 4 dots). Interestingly, under the joint evolution of dispersal and 
400

401

402

403

404

405

406

407

408

409

410

411

412

413

414

415

416

417

418

419

420

421

422

to when evolving under fix low dispersal (Fig. 1D-F). Specifically, for a given dispersal cost, higher polyandry (lower strength of direct selection on female re-mating) led to the accumulation of lower inbreeding load in offspring survival, especially for lower costs of dispersal (Fig. 4, dots; Table S3). The difference was especially evident when comparing no cost vs costly polyandry. For the inbreeding load in adult reproduction, this effect was present but only very slight and with overlapping confidence intervals between different costs of polyandry (Fig. 4, diamonds; Table S3). This result is perhaps surprising and counterintuitive as the expectation would be for more polyandry to lead to accumulation of higher load, due to reduced inbreeding and purging, and not vice versa as observed here. Finally, the emerging heterosis in the system was exclusively driven by the level of evolved dispersal, and hence by dispersal cost. Higher heterosis was present under high costs of dispersal, thus generating strong positive selection for dispersal, counteracting the high cost (Fig. 4B).

A

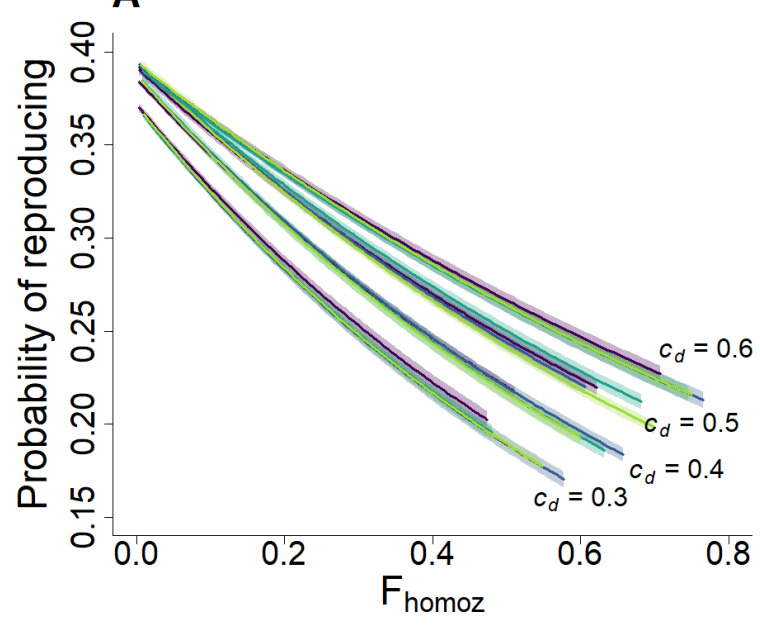

B

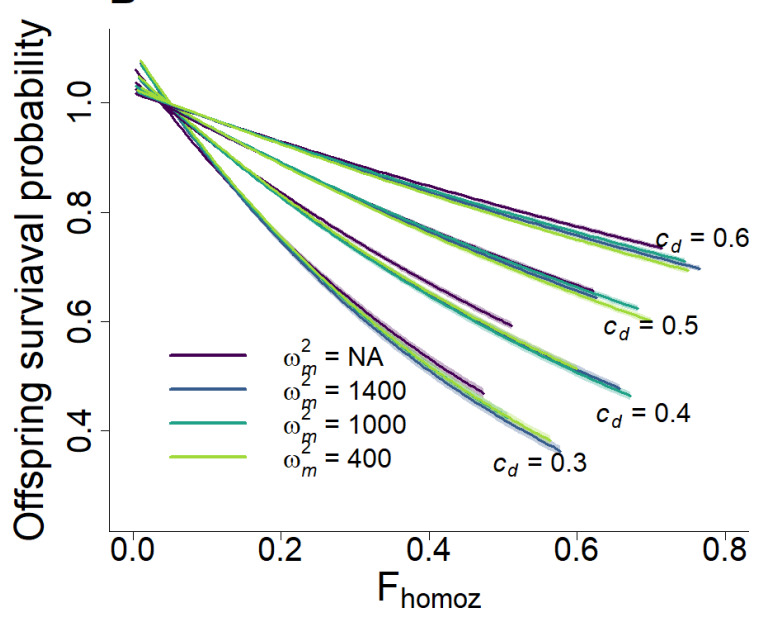

Figure 3. Jointly evolving dispersal and polyandry affect evolution of inbreeding depression in reproduction and survival. Relationship between $\mathbf{A}$ ) individual probability of reproducing and inbreeding coefficient $F_{\text {homoz }}$ (i.e., inbreeding depression in reproduction probability), and B) between offspring survival probability and $F_{\text {homoz }}$ (i.e., inbreeding depression in offspring survival probability). Results are presented for varying costs of dispersal $\left(c_{d}\right)$ and strengths of direct selection against female multiple mating $\left(\omega_{m}^{2}\right.$; colors). Lines show the fitted models and coloured shades the $95 \% \mathrm{Cl}$. The coefficients (i.e., mutation load and inbreeding load) and standard errors of all the fitted models are presented in Table S3. Models are fitted to simulations results at generation 20,000 to a subsample of 140 populations, across 10 replicates. 

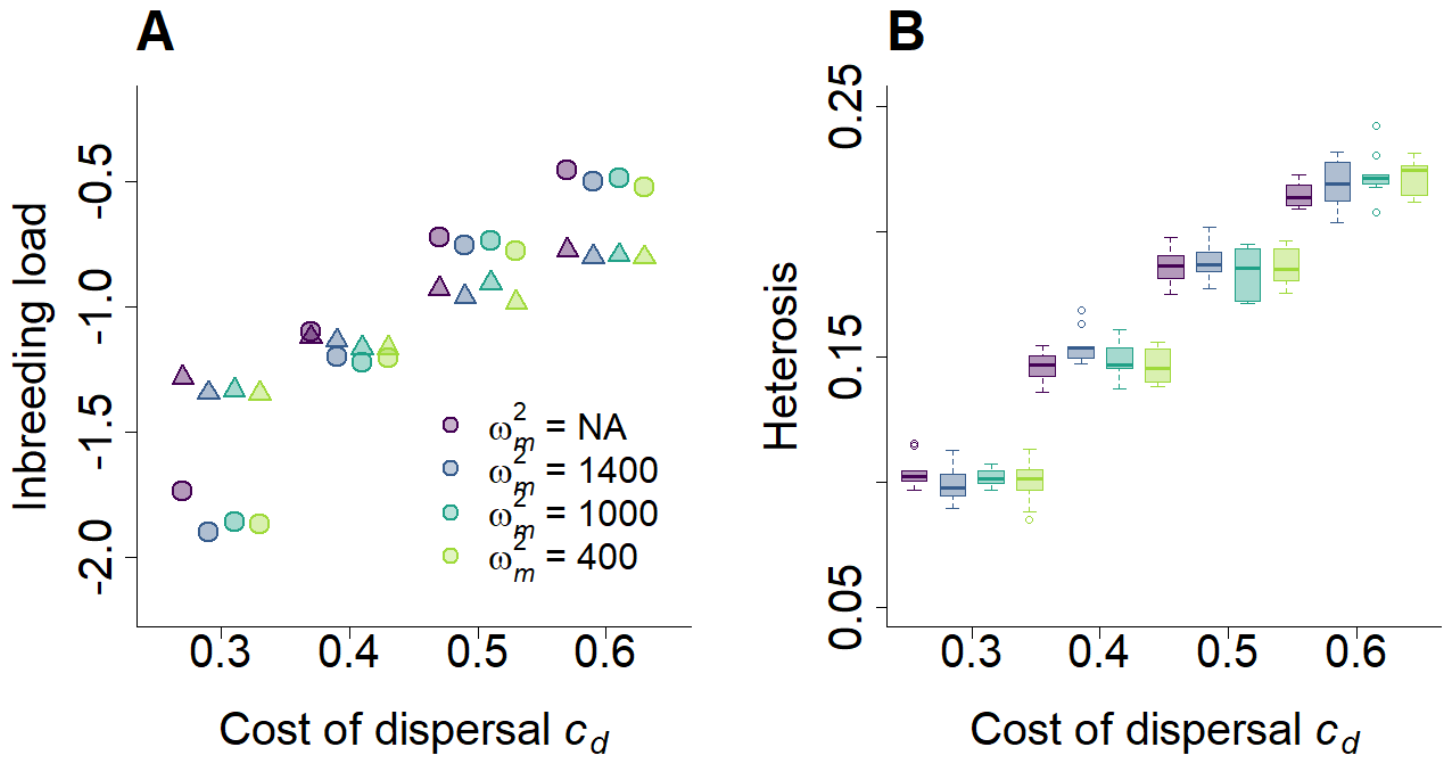

Figure 4. Inbreeding load and heterosis emerging under the joint evolution of dispersal and polyandry. A) Estimated slopes of probability of offspring survival (dots) and probability of reproduction (triangles) on individual $\mathrm{F}_{\text {homoz }}$ (i.e., inbreeding load), for different cost of dispersal $\left(c_{d}\right)$ and strengths of direct selection on female multiple mating ( $\omega_{m}^{2}$; colors). Results are on the log scale. Standard errors are not shown because smaller than the dots size. Models are fitted to simulations results at generation 20,000 to a subsample of 140 populations, across 10 replicates. B) Heterosis as a function of $c_{d}$ and $\omega_{m}^{2}$ (the color legend is the same as in A). Heterosis is shown as median (solid bands), first and third quartiles (box limits), and approximately twice the standard deviation (whiskers) over 20 replicate simulations at generation 20,000 .

General discussion. These results shed light on the previously unconsidered intimate connection existing between dispersal and polyandry evolution through their shared driver of inbreeding depression, and on their effect on evolution of inbreeding depression itself. They thereby highlight important interactions between the evolution of two fundamental life-histories I behaviours. Specifically, I showed that through their effect on the population relatedness structure, and hence on inbreeding levels, dispersal and female multiple mating affect each other evolution in spatially structured populations. The engine of this feedback is inbreeding depression. Without genetic load giving rise to inbreeding depression and heterosis, kin competition alone is not sufficient for a negative relationship between dispersal and polyandry to emerge. 
Acknowledgments. I am grateful to J. M. Reid and J. M. J. Travis as this work would not have

been possible without their mentorship. I thank A. B. Duthie, M. Tschol, A. Charmouh and L.

Dunan for helpful discussions. This work was supported by a Royal Society University

Research Fellowship to GB (UF160614). All simulations were performed on the University of

Aberdeen HPC, Maxwell.

\section{References}

Agrawal, A. F., and M. C. Whitlock. 2011. Inferences about the distribution of dominance drawn from yeast gene knockout data. Genetics 187:553-566.

Bocedi, G., and J. M. Reid. 2017. Feed-backs among inbreeding, inbreeding depression in sperm traits, and sperm competition can drive evolution of costly polyandry. Evolution 71:2786-2802.

Bonte, D., H. van Dyck, J. M. Bullock, A. Coulon, M. Delgado, M. Gibbs, V. Lehouck, E. Matthysen, K. Mustin, M. Saastamoinen, N. Schtickzelle, V. M. Stevens, S. Vandewoestijne, M. Baguette, K. A. Bartoń, T. G. Benton, A. Chaput-Bardy, J. Clobert, C. Dytham, T. Hovestadt, C. M. Meier, S. C. F. Palmer, C. Turlure, and J. M. J. Travis. 2012. Costs of dispersal. Biological Reviews of the Cambridge Philosophical Society 87:290-312.

Caballero, A., and P. D. Keightley. 1994. A pleiotropic nonadditive model of variation in quantitative traits. Genetics 138:883-900.

Charlesworth, D., and B. Charlesworth. 1987. Inbreeding depression and its evolutionary consequences. Annual Review of Ecology and Systematics 18:237-268.

Charlesworth, D., and J. H. Willis. 2009. The genetics of inbreeding depression. Nature Reviews. Genetics 10:783-96.

Clobert, J., M. Baguette, T. G. Benton, and J. M. Bullock. 2012. Dispersal Ecology and Evolution. Oxford University Press.

Cornell, S. J., and T. Tregenza. 2007. A new theory for the evolution of polyandry as a means of inbreeding avoidance. Proceedings of the Royal Society B 274:2873-9.

Cote, J., E. Bestion, S. Jacob, J. Travis, D. Legrand, and M. Baguette. 2017. Evolution of dispersal strategies and dispersal syndromes in fragmented landscapes. Ecography 40:56-73.

Duthie, A. B., G. Bocedi, R. R. Germain, and J. M. Reid. 2018. Evolution of precopulatory and post-copulatory strategies of inbreeding avoidance and associated polyandry. Journal of Evolutionary Biology 31:31-45. 
Duthie, A. B., G. Bocedi, and J. M. Reid. 2016. When does female multiple mating evolve to adjust inbreeding? Effects of inbreeding depression, direct costs, mating constraints, and polyandry as a threshold trait. Evolution 70:1927-1943.

Eyre-Walker, A., and P. D. Keightley. 2007. The distribution of fitness effects of new mutations. Nature reviews. Genetics 8:610-8.

Fromhage, L., H. Kokko, and J. M. Reid. 2009. Evolution of mate choice for genome-wide heterozygosity. Evolution 63:684-94.

Gandon, S. 1999. Kin competition, the cost of inbreeding and the evolution of dispersal. Journal of Theoretical Biology 200:345-364.

Germain, R. R., P. Arcese, and J. M. Reid. 2018. The Consequences of Polyandry for Sibship Structures, Distributions of Relationships and Relatedness, and Potential for Inbreeding in a Wild Population. The American Naturalist 191:638-657.

Gilbert, K. J., N. P. Sharp, A. L. Angert, G. L. Conte, J. A. Draghi, F. Guillaume, A. L. Hargreaves, R. Matthey-Doret, and M. C. Whitlock. 2017. Local Adaptation Interacts with Expansion Load during Range Expansion: Maladaptation Reduces Expansion Load. The American Naturalist 189:368-380.

Guillaume, F., and N. Perrin. 2009. Inbreeding load, bet hedging, and the evolution of sexbiased dispersal. The American Naturalist 173:536-41.

Guillaume, F., and N. Perrin. 2006. Joint evolution of dispersal and inbreeding load. Genetics 173:497-509.

Hedrick, P. W., and A. Garcia-Dorado. 2016. Understanding Inbreeding Depression, Purging, and Genetic Rescue. Trends in Ecology \& Evolution 31:940-952. Elsevier Ltd.

Henry, R. C., A. Coulon, and J. M. J. Travis. 2016. The evolution of male-biased dispersal under the joint selective forces of inbreeding load and demographic and environmental stochasticity. The American Naturalist 188:423-433.

Huber, C. D., A. Durvasula, A. M. Hancock, and K. E. Lohmueller. 2018. Gene expression drives the evolution of dominance. Nature Communications 9. Springer US.

Jennions, M. D., and M. Petrie. 2000. Why do females mate multiply? A review of the genetic benefits. Biological Reviews 75:21-64.

Keller, L., and D. Waller. 2002. Inbreeding effects in wild populations. Trends in Ecology \& Evolution 17:19-23.

Kimura, M. 1965. A stochastic model concerning the maintenance of genetic variability in quantitative characters. Proceedings of the National Academy of Sciences 54:731-736.

Lande, R., and D. Schemske. 1985. The evolution of self-fertilization and inbreeding depression in plants. I. Genetic models. Evolution 39:24-40.

Lande, R., D. W. Schemske, and S. T. Schultz. 1994. High inbreeding depression, selective interference among loci, and the threshold selfing rate for purging recessive lethal mutations. Evolution 48:965-978.

Legrand, D., J. Cote, E. A. Fronhofer, R. D. Holt, O. Ronce, N. Schtickzelle, J. M. J. Travis, and J. Clobert. 2017. Eco-evolutionary dynamics in fragmented landscapes. Ecography 40:9-25. Blackwell Publishing Ltd. 
Li, X. Y., and H. Kokko. 2019. Sex-biased dispersal: a review of the theory. Biological Reviews 94:721-736.

Markert, J. A., P. R. Grant, B. R. Grant, L. F. Keller, J. L. Coombs, and K. Petren. 2004. Neutral locus heterozygosity, inbreeding, and survival in Darwin's ground finches (Geospiza fortis and G. scandens). Heredity 92:306-315.

Morton, N. E., J. F. Crow, and H. J. Muller. 1956. An estimate of the mutational damage in man from data on consanguineous marriages. Proceedings of the National Academy of Sciences of the United States of America 42:855-863.

Neff, B. D., and T. E. Pitcher. 2008. Mate choice for non-additive genetic benefits: a resolution to the lek paradox. Journal of Theoretical Biology 254:147-55.

Nietlisbach, P., S. Muff, J. M. Reid, M. C. Whitlock, and L. F. Keller. 2019. Nonequivalent lethal equivalents: Models and inbreeding metrics for unbiased estimation of inbreeding load. Evolutionary Applications 12:266-279. Wiley-Blackwell.

Peischl, S., M. Kirkpatrick, and L. Excoffier. 2015. Expansion Load and the Evolutionary Dynamics of a Species Range. The American Naturalist 185:E81-E93.

Perrin, N., and J. Goudet. 2001. Inbreeding, kinship, and the evolution of natal dispersal. Pp. 123-142 in J. Clobert, E. Danchin, A. A. Dhondt, and J. D. Nichols, eds. Dispersal. Oxford University Press, Oxford.

Perrin, N., and V. Mazalov. 1999. Dispersal and inbreeding avoidance. The American Naturalist 154:282-292.

Perrin, N., and V. Mazalov. 2000. Local competition, inbreeding, and the evolution of sexbiased dispersal. The American Naturalist 155:116-127.

Pizzari, T., and N. Wedell. 2013. The polyandry revolution. Philosophical Transactions of the Royal Society B 368:20120041.

Porcher, E., and R. Lande. 2016. Inbreeding depression under mixed outcrossing, selffertilization and sib-mating. BMC Evolutionary Biology 16:105. BMC Evolutionary Biology.

Porcher, E., and R. Lande. 2005. Loss of gametophytic self-incompatibility with evolution of inbreeding depression. Evolution 59:46-60.

Ravigné, V., I. Olivieri, S. Gonzalez-Martinez, and F. Roussett. 2006. Selective Interactions Between Short-Distance Pollen and Seed Dispersal in Self-Compatible Species. Evolution 60:2257-2271.

R Core Team. 2019. R: A Language and Environment for Statistical Computing. R Foundation for Statistical Computing, Vienna, Austria.

Reid, J. M., P. Arcese, L. F. Keller, R. R. Germain, A. B. Duthie, S. Losdat, M. E. Wolak, and P. Nietlisbach. 2015. Quantifying inbreeding avoidance through extra-pair reproduction. Evolution 69:59-74.

Reid, J. M., and R. J. Sardell. 2012. Indirect selection on female extra-pair reproduction? Comparing the additive genetic value of maternal half-sib extra-pair and within-pair offspring. Proceedings of the Royal Society B 279:1700-8.

Ronce, O. 2007. How Does It Feel to Be Like a Rolling Stone? Ten Questions About Dispersal Evolution. Annual Review of Ecology, Evolution, and Systematics 38:231-253. 
Roze, D., and F. Rousset. 2009. Strong effects of heterosis on the evolution of dispersal rates. Journal of Evolutionary Biology 22:1221-1233.

Schultz, S. T., and M. Lynch. 1997. Mutation and Extinction: The Role of Variable Mutational Effects, Synergistic Epistasis, Beneficial Mutations, and Degree of Outcrossing. Evolution 51:1363-1371.

Simmons, M. J., and J. F. Crow. 1977. Mutations affecting fitness in Drosophila populations. Annu Rev Genet 11:49-78.

Spigler, R. B., K. Theodorou, and S.-M. Chang. 2017. Inbreeding depression and drift load in small populations at demographic disequilibrium. Evolution 71:81-94.

Szulkin, M., K. Stopher, J. Pemberton, and J. Reid. 2013. Inbreeding avoidance, tolerance, or preference in animals? Trends in Ecology \& Evolution 28:205-211.

Taylor, M. L., T. A. R. Price, and N. Wedell. 2014. Polyandry in nature: a global analysis. Trends in Ecology \& Evolution 29:376-83. Elsevier Ltd.

Theodorou, K., and D. Couvet. 2006. On the expected relationship between inbreeding, fitness, and extinction. Genetics Selection Evolution 38:371-387.

Tregenza, T., and N. Wedell. 2002. Polyandrous females avoid costs of inbreeding. Nature 415:71-73.

Waser, P., S. Austad, and B. Keane. 1986. When should animals tolerate inbreeding? The American Naturalist 128:529-537.

Whitlock, M. C., P. K. Ingvarsson, and T. Hatfield. 2000. Local drift load and the heterosis of interconnected populations. Heredity 84:452-457. 